\title{
New Researches on the Role of Virtual Exhibitions in Digitization, Preservation and Valorization of Cultural Heritage
}

\author{
Cristian CIUREA ${ }^{1}$, Florin Gheorghe FILIP ${ }^{2}$ \\ ${ }^{1}$ Bucharest University of Economic Studies, Bucharest, Romania \\ ${ }^{2}$ Romanian Academy - INCE \& BAR, Bucharest, Romania \\ cristian.ciurea@ie.ase.ro,ffilip@acad.ro
}

The current paper presents a scientific approach of implementing online virtual exhibitions on mobile devices in order to increase the number of visitors inside cultural institutions, such as galleries, libraries, archives and museums (GLAM). There are described the new opportunities offered by the cultural heritage to generate revenues for cultural institutions and to contribute at the economic development of the belonging countries. It is described the role of virtual exhibitions in the processes of digitization, preservation and valorization of cultural heritage. The most important features of virtual exhibitions, which makes the differences from simple image galleries and websites, are presented and detailed.

Keywords: Cultural Heritage, Virtual Exhibitions, Mobile Applications, Digitization.

\section{1} Introduction

Nowadays, we are experiencing a technological revolution and every human activity is influenced by the new ICT. The modern information and communication technologies that we are using today, such as mobile technologies, cloud computing, Internet of Things (IoT) and so on, have impacted our lives and our fields of activity. The cultural sector needs to adapt to this (r)evolution, because people are not coming anymore in a library to read a book in physical format if they have the possibility to read it online. This happens also in the case of museums and art galleries, where visitors don't come anymore to see a painting, a sculpture or other cultural object, and they are satisfied with an online image of that cultural object that they find on Google or other place on the web.

The current society presents a different behavior than the society of ten or twenty years ago. This should be taken into account when deciding the application of a strategy to increase the number of visitors or the revenues of cultural institutions.

According to the Cultural Barometer in 2015, $38 \%$ of people analyzed have not read any books in the last year and only $16 \%$ said they participate often at cultural events, theater, museums and art exhibitions, cinema, opera, philharmonic and entertainment performances. From the young people aged between 14 and 30 years, $48 \%$ said that they go to museums and exhibitions, and only $35 \%$ goes to the library.

A modern form of presentation of cultural objects through which is enhanced their security and which creates the premises for an easier access at any time and from any point from the globe is that of online virtual exhibitions (OLVE). An OLVE is not just "another way" to present cultural objects using a new technology. Through its dynamic character (it can be updated at any time) and the variety of content and forms of presentation, an OLVE is more than a digitized collection or an eBook.

As defined in [11], a "virtual exhibition translates the essence of the physical objects presented into the digital world and makes this essence available to users anywhere, anytime effectively and efficiently."

The objective of virtual exhibitions is to attract the visitors to come back in a cultural institution to see with their eyes the real cultural object and to not be satisfied only with a simple picture of that object. The development and implementation of virtual exhibitions have the first objective to promote and valorize the cultural heritage from cultural institutions and after that to achieve the other particular objectives. 
A virtual exhibition allow the visitors to try alternative routes for navigation through the content and also, it offers a way to achieve possibilities that visitors were not able to do in a real exhibition [9].

When we discuss about the implementation of virtual exhibitions, we must take into consideration the previous scientific literature and all the recommendations from people that have worked in the cultural sector and have created and curated physical exhibitions [1], [2], [3]. A virtual exhibition is not only an image gallery where the user can scroll to see the pictures inside the gallery. The virtual exhibition needs to interact with the users, to collect their experiences, to give them what they need, in order to determine them to visit the physical exhibition or a number of the cultural objects that they found inside the virtual exhibition.

In Figure 1 below is presented the expected evolution of number of visitors in a cultural institution, correlated with the number of virtual exhibitions implemented with cultural objects of that institution.

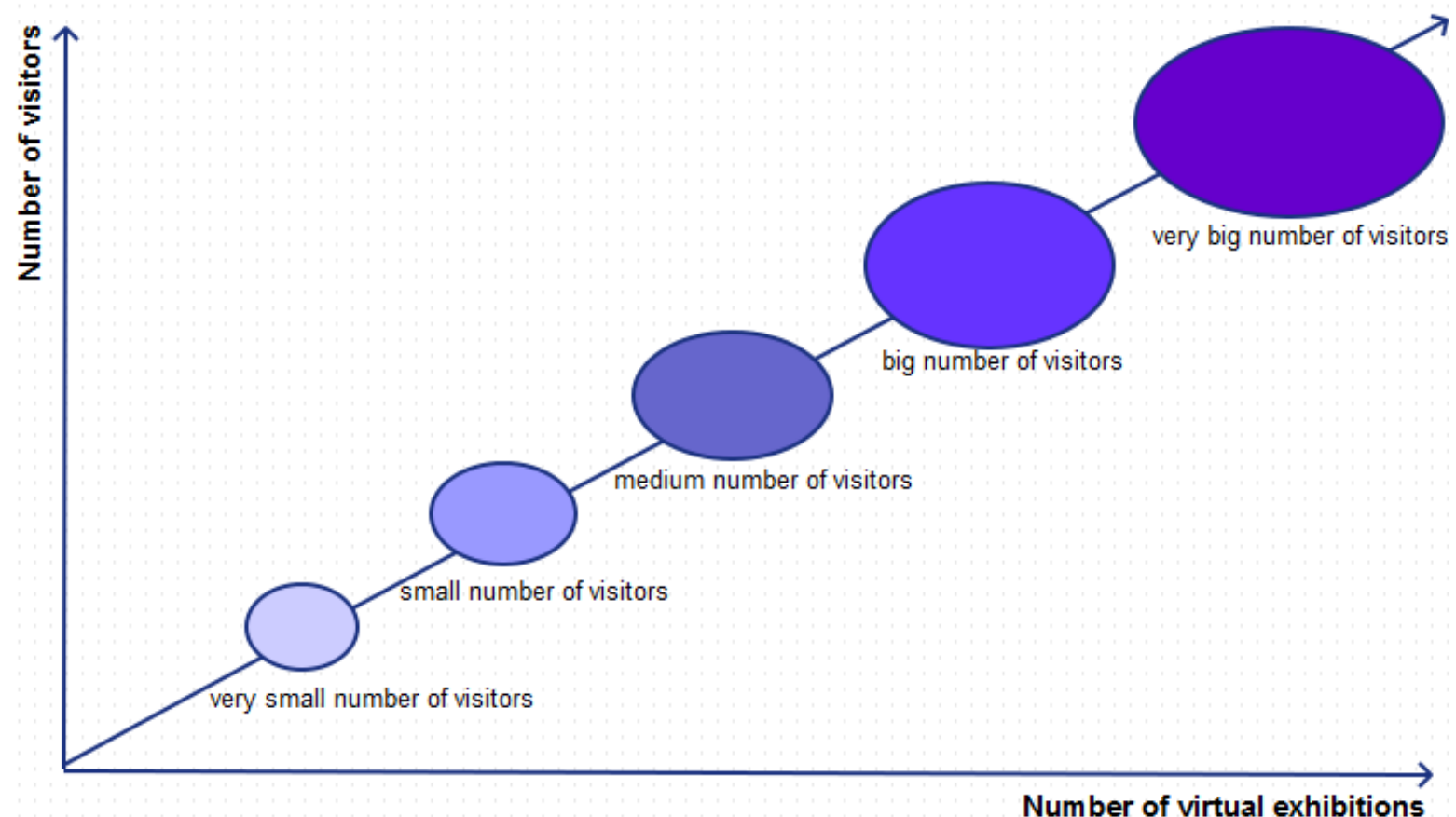

Fig. 1. The evolution of number of visitors based on the number of virtual exhibitions

As it can be seen from Figure 1, the number of visitors will grow if the number of virtual exhibitions will increase. This hypothesis needs to be validated, starting with few cultural institutions that agree to implement and make available to the public some virtual exhibitions and to collect data about their number of visitors. Regarding the number of visitors, it is very difficult to have accurate information about the number of unique visitors during a period of time, because there are some visitors that come back many times to visit the institution. We can have details about the number of visits, but not about the number of unique visitors [4].
The main reason of online virtual exhibitions (OLVE) implementation is to attract visitors to come in a cultural institution to see for themselves the real cultural objects and not be satisfied only with a simple online image of a cultural object, like a painting, sculpture, manuscript or an old book.

The virtual exhibition needs users' interaction to collect their experiences, to give them what they need, to get them to visit the real exhibition or only a number of cultural objects that they have found inside the virtual exhibition.

Virtual exhibitions can be implemented both in the online version, being available on the Internet from any computer or mobile device, 
and also in the version of mobile applications, being developed natively for mobile operating systems of smartphones and tablets.

Virtual exhibitions have some advantages such as the possibility of reuse, the minimization of security risks for original items and reduction of costs when the exhibition is meant to be performed in different locations from those of preserving objects.

The most important characteristics of virtual exhibitions are the following:

- virtual exhibitions can replace the physical guide of real exhibitions, meaning that they represents a substitute of the catalogue that describe the collections;

- virtual exhibitions can include multimedia content, such as videos, animations, dynamic galleries, which demonstrate that a virtual exhibition is not only a simple image gallery or a website for presentation of cultural heritage collections;

- virtual exhibitions can be implemented as native mobile applications that widen the possibilities for making accessible cultural heritage collections on mobile devices, such as smartphones and tablets. Taking into account the above statements, a virtual exhibition is a concept that has acquired new meanings along with the evolution of modern information and communication technologies.

The virtual exhibition represents a new research field of the e-Family category, and it has less theoretical background to indicate its research and development direction. The development of a modeling framework for virtual exhibitions is very important for IT managers and designers in this field [10].

\section{Implementation Examples of Virtual Exhibitions}

The implementation of a virtual exhibition implies the participation of several categories of specialists from various fields (ICT, libraries, museums, and so on).

The implementation of virtual exhibitions for mobile devices can be realized as:
- web applications, available both from PCs and mobile devices; in this case, the application must have an adaptive interface, meaning to change depending on screen size and resolution in order to enable a better user experience;

- native mobile applications created specifically for mobile devices with a particular operating system, such as Android, iOS or Windows Phone; in this case, the application installs and runs only on mobile devices with the operating system for which it was developed.

Both implementations are effective and each variant offers some advantages. For example, web applications allow access from any type of device, including the personal computer. Native mobile applications offers advantages regarding the access to hardware features of the mobile device, such as the GPS or camera, which enable scanning of QR (Quick Response) codes when the user is located inside the physical exhibition or the possibility for localization on the map of the cultural objects.

The Romanian Academy Library was involved in the AthenaPlus project (http://athenaplus.eu) and it was the beneficiary of an exciting tool designed to create virtual exhibitions, named MOVIO (http://wiki.athenaplus.eu/index.php/MOVIO ) [5]. This tool allows to create online virtual exhibitions that can be exported as native mobile applications for mobile devices with iOS and Android.

The main advantage of implementing a virtual exhibition for mobile devices, even if it is a responsive web application or a native mobile application, is that it can be accessed anytime and from anywhere, because of the portability feature offered by a tablet or smartphone. This characteristic was the key for the explosive evolution of mobile devices market.

During the last years, the Romanian Academy Library implemented three virtual exhibitions with MOVIO, namely:

- Constantin Brancoveanu - prince of Wallachia, which can be accessed at: http://movio.biblacad.ro/BRANCO/ 
- Greek Coins - Constantin Orghidan collection of the Romanian Academy Library, which can be accessed at: http://movio.biblacad.ro/COINS/

- Seals - the history treasure, which is available

http://movio.biblacad.ro/SEALS/

- S,tefan Luchian - illustrator, available at: http://movio.biblacad.ro/LUCHIAN/

- Saint Antim Ivireanul, which can be accessed http://movio.biblacad.ro/ANTIM/

- To other worlds - painting exhibition of Vasilica CHIFU, which is available at: http://movio.biblacad.ro/CHIFU/
The Figure 2 below presents the mobile versions of the first three virtual exhibitions created with MOVIO and implemented at the Romanian Academy Library, the provider of cultural objects used in the implementation. The figure presents a screen capture from the main page of the following virtual exhibitions: Constantin Brancoveanu - prince of Wallachia, Greek Coins - Constantin Orghidan collection of the Romanian Academy Library and Seals - the history treasure.

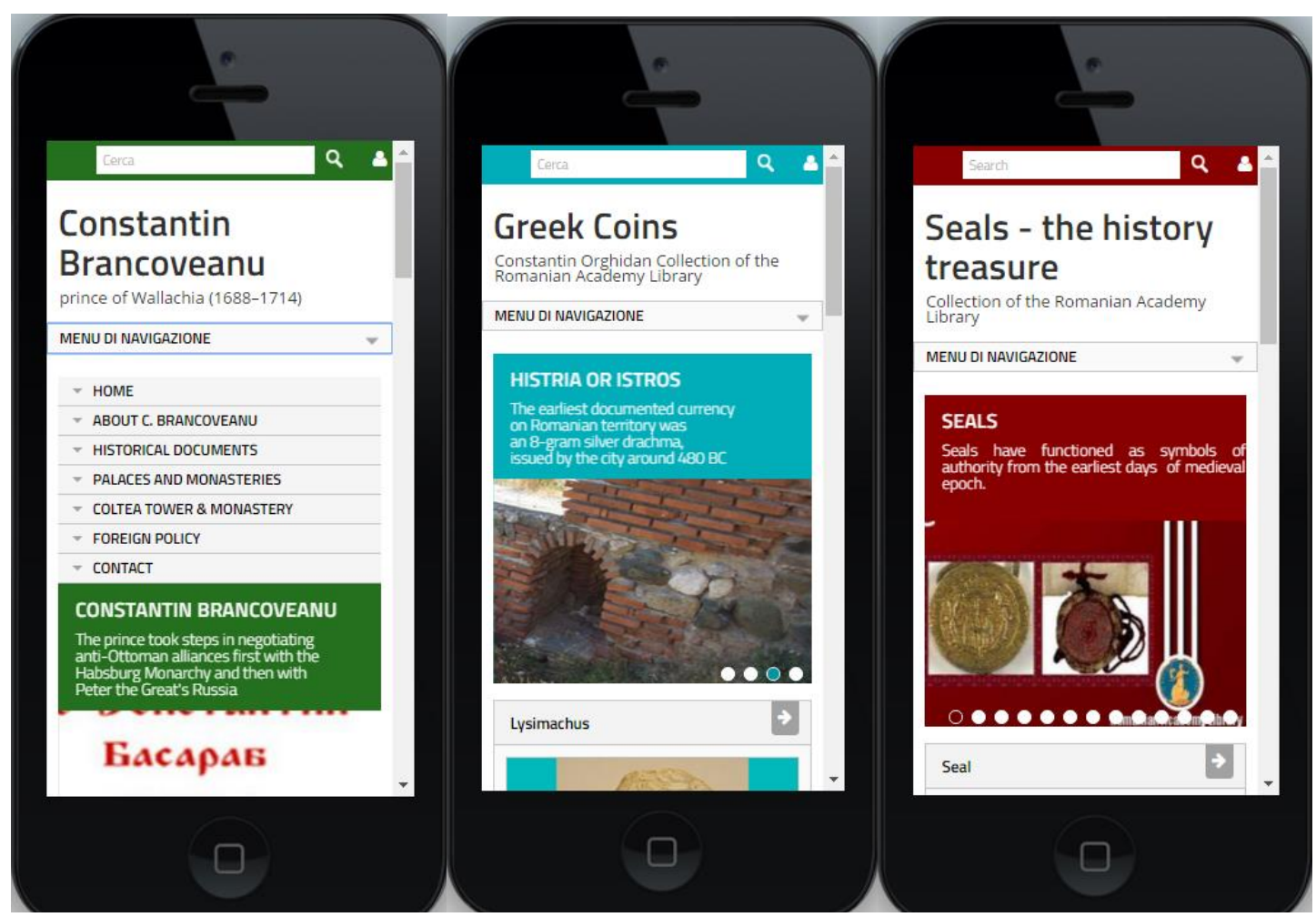

Fig. 2. The virtual exhibitions implemented with MOVIO

The MOVIO tool is a semantic content management system that enables users to create their own ontologies and use them immediately without having specialized knowledge in the ICT field.
Figure 3 below presents a screen capture from the following virtual exhibitions: Ștefan Luchian - illustrator, Saint Antim Ivireanul and To other worlds - painting exhibition of Vasilica CHIFU. 


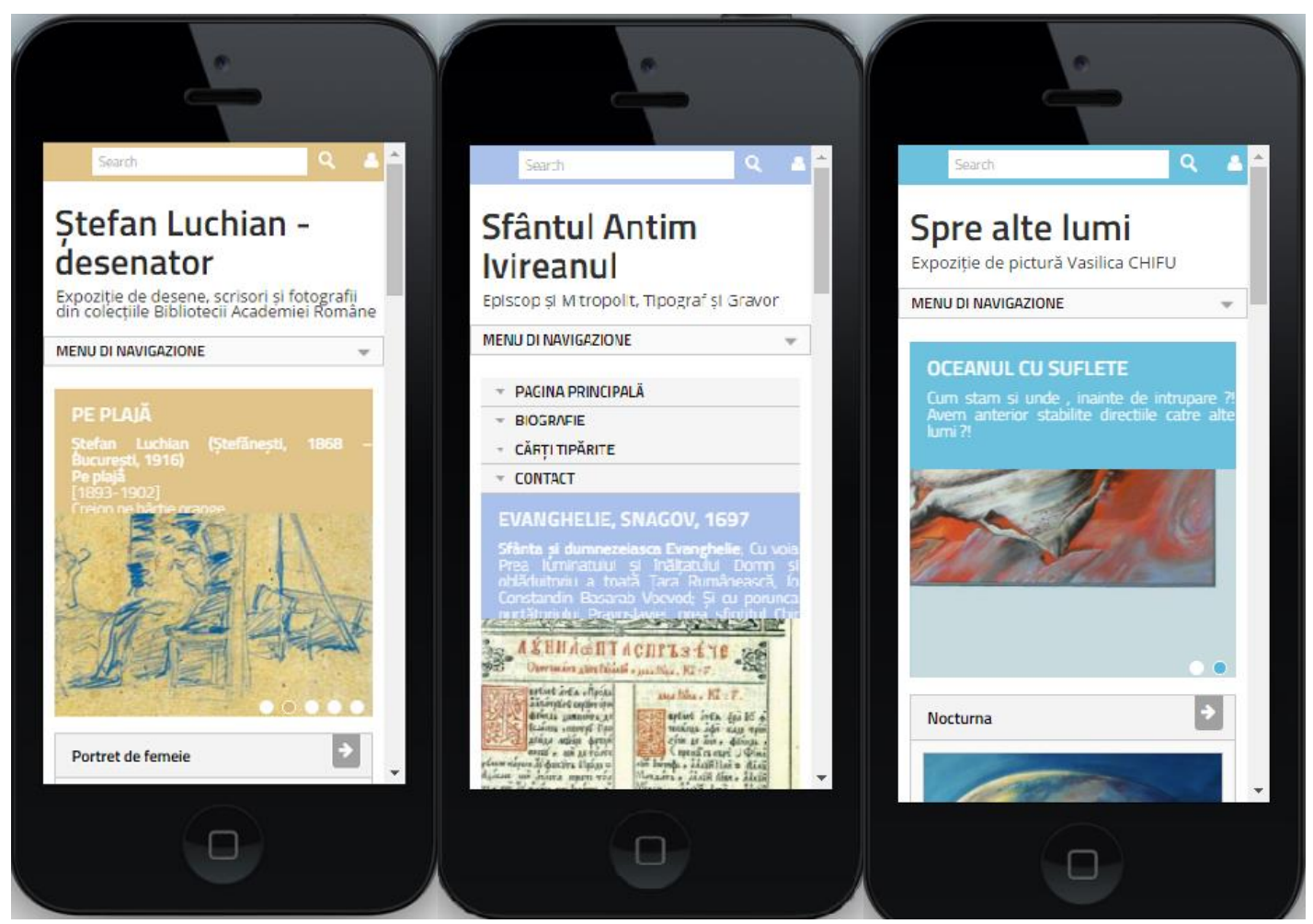

Fig. 3. MOVIO implementations of virtual exhibitions

The implementation of virtual exhibition using scanned cultural objects belonging to a cultural institution or to many cultural institutions is not a simple process. There arise problems regarding the copyright, permissions, pictures resolutions and so on. Excepting these technical problems, we must surpass the mentality of people from cultural institutions, who think that if they will make available online a digital copy of cultural object, then visitors will not be interested to come inside the institution to access that object. In this way, the managers of cultural institutions, such as libraries and museums, are afraid that their institutions will lose the current visitors in the near future. This hypothesis was contradicted by different examples of museums that sells digital copies of famous paintings (for example Monalisa or Gioconda of Leonardo Da Vinci), and their number of visitors has increased [6].

It seems that the marketing strategies are working also in the cultural heritage area and cultural institutions needs to better promote their collections in order to attract many visitors. Some of the young people doesn't know which are the opportunities offered by a museum or library besides that pass daily on their way. The role of virtual exhibitions is to open the mind of the target public and to tell them what they can see.

\section{Digitization, Preservation and} Valorization of Cultural Heritage

Europe has realized that it possess a rich cultural heritage which could be exploited not only for cultural reason, but also in economic purposes. The cultural heritage is considered as a strategic economic resource that can generate income for cultural institutions.

Many European institutions have reconsidered the cultural heritage in the last years and have discovered the potential that cultural heritage can offer for the economic development of institutions and countries that manage this cultural heritage. The cultural heritage is considered as a strategic economic resource which can generate revenues for cultural institutions [7]. The problem is that the big part of the cultural heritage available in libraries, archives and museum is not digitized. At this moment, only $10 \%$ of the European cultural heritage have been digitized and the rest of $90 \%$ needs to be digitized in the following years. This process will require a lot of resources that must be 
allocated by cultural institutions or by government in the next period.

As mentioned in [8], cultural institutions are interested in the digitization process of their collections not only for the preservation of the cultural heritage, but also to make the collections available to the wider public in a way that can be attractive.

In order to achieve the digitization, preservation and valorization of cultural heritage, we need to consider the following processes:

- the digitization must be realized by scanning or photographing the cultural objects and the assignment of metadata, in order to create accurate databases with digital formats of cultural objects;

- the preservation of digitized objects means to ensure the high quality of digital formats of cultural objects and to make backups for safety;

- the implementation of virtual exhibitions means to design, develop, deploy and maintain virtual exhibitions, in order to promote different collections, to attract visitors and to valorize the cultural heritage.

Figure 4 below shows all the three processes and their aggregated output, which influence the cultural heritage.

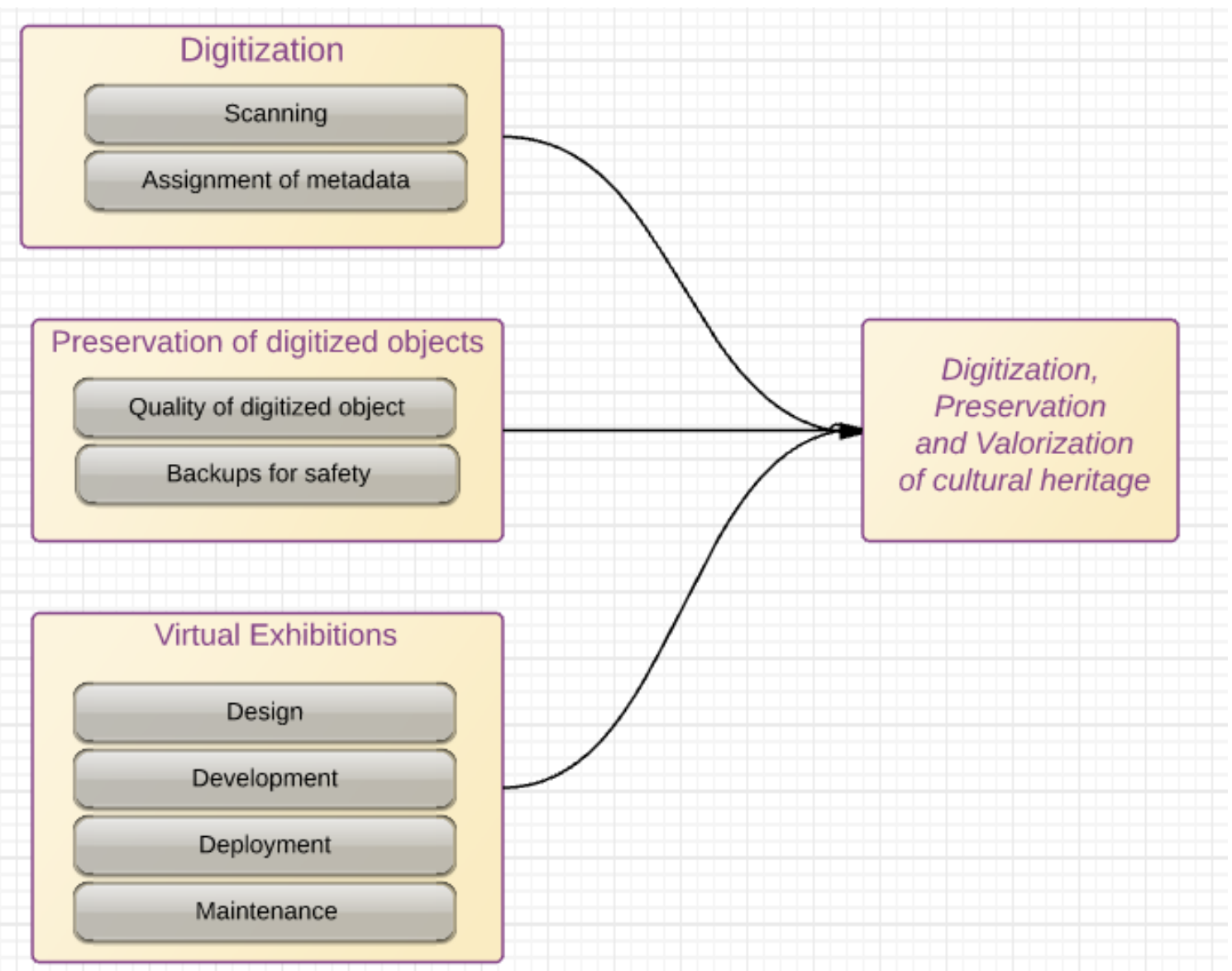

Fig. 4. The digitization, preservation and valorization of cultural heritage

Digitization of cultural heritage collections is regarded as a major concern of the European institutions. The advantages to transform physical objects into digital objects are obvious: it prevents their destruction, it offers opportunities that did not exist before.

Digitized collections can be used also in various commercial services. The Europeana portal (http://www.europeana.eu) owns more than 53 million cultural artifacts in digital format and represents a huge potential as a valued resource for the educational sector.

The virtual exhibitions have a significant influence on the above processes, namely the digitization and preservation of digitized objects, because if these processes deliver 
better outputs, then the design and development of virtual exhibitions will be easier. If we have many digitized objects, we can choose between them, in order to create the most attractive virtual exhibitions that can determine visitors to come into the cultural institutions.

\section{Conclusions}

The present day society shows a different behavior than the society from ten or twenty years ago. This aspect must be taken into consideration when we decide to apply a strategy to increase the number of visitors or the revenues of cultural institutions. The marketing policies have an extraordinary impact on human behavior and this must be exploited in order to promote the culture among the young people, especially. The development and implementation of virtual exhibitions has the first objective to promote and valorize the cultural heritage from cultural institutions. After that, the other objectives will be carried out by default, including the number of visitors increasing and the increase of cultural institutions revenues.

By involving specialists and computer scientists to bring new information technologies in the cultural sector will ensure the development of cultural institutions, both culturally and in economic terms.

\section{Acknowledgement}

The current paper is an extended version of the work presented at the 15th International Conference on Informatics in Economy, IE 2016, 02 - 05 June 2016, Cluj-Napoca, Romania [12].

\section{References}

[1] F. G. Filip, C. Ciurea, H. Dragomirescu, I. Ivan, "Cultural Heritage and Modern Information and Communication Technologies," Technological and Economic Development of Economy, Vol. 21, No. 3, May 2015, Taylor \& Francis, pp. 441-459.

[2] C. Ciurea, F. G. Filip, "A Business Model for the Interaction Between Actors of Cultural Economy," Proceedings of the 14th International Conference on Informatics in Economy, IE 2015, 30 April - 03 May 2015, Bucharest, Romania, ASE Printing House, ISSN 2284-7472, ISSN-L 2247-1480.

[3] F. G. Filip, "Information Technologies in Cultural Institutions," Studies in Informatics and Control, Vol. 6, No. 4, 1996, pp. 385-400.

[4] C. Ciurea, F. G. Filip, "Validation of a Business Model for Cultural Heritage Institutions," Informatica Economică, Vol. 19, No. 2, 2015, ISSN 1453-1305, pp. 4656.

[5] M. T. Natale, S. H. Minelli, B. Dierickx, P. Ongaro, M. Piccininno, D. Ugoletti, R. Saccoccio, A. Raggioli, "Exhibiting Intangible Cultural Heritage using MOVIO: a multilingual toolkit for creating curated digital exhibitions, made available by the AthenaPlus project," ICOM 2014 Access and Understanding - Networking in the Digital Era: intangible Cultural Heritage, 2014, Available at: http://www.cidoc2014.de/images/sampled ata/cidoc/papers/H-2_Natale_Minelli_etal_paper.pdf

[6] M. Hagedorn-Saupe, A. Peukert, „New Ways of Presenting the Past | Work, Research, and Findings of the International Digital Exhibitions Working Group“, Uncommon Culture, Vol. 6, No. 1 (11), 2015, ISSN 2083-0599 (online), 20826923 (print), pp. 37-42.

[7] EC 2015, Getting cultural heritage to work for Europe, Report of the Horizon 2020 Expert Group on Cultural Heritage, 2015, ISBN 978-92-79-46046-3, Available at: http://www.kowi.de/Portaldata/2/Resource s/horizon2020/coop/H2020-ReportExpert-Group-Cultural-Heritage.pdf

[8] S. Styliani, L. Fotis, K. Kostas, P. Petros, "Virtual museums, a survey and some issues for consideration", Journal of Cultural Heritage, No. 10, 2009, pp. 520528.

[9] G. Lepouras, A. Katifori, C. Vassilakis, D. Charitos, "Real Exhibitions in a Virtual Museum", Virtual Reality, Vol. 7, Issue 2, 2004, pp 120-128 
[10] H. Ling, D. Dai, "A Modeling Framework for Virtual Exhibition," 2010 International Conference on Management and Service Science, Wuhan, 2010, pp. 15.

[11] L. Corovic, V. Vuksan, A. Sofronijevic, "Virtual exhibition as a medium for presenting scientific and cultural heritage to international audiences: Dorde Stanojevic - a rector who lit up Belgrade", The 9th SEEDI Conference: Digitisation of cultural and scientific heritage, Belgrade, Serbia, 15th - 16th May, 2014.

[12] C. Ciurea, F. G. Filip, "The Role of Virtual Exhibitions in Cultural Heritage Digitization, Preservation and Valorization," Proceedings of the 15th International Conference on Informatics in Economy, IE 2016, 02 - 05 June 2016, Cluj-Napoca, Romania.

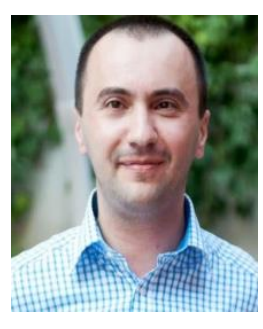

Cristian CIUREA is Associate Professor at the Department of Economic Informatics and Cybernetics from Bucharest University of Economic Studies. He has graduated the Faculty of Economic Cybernetics, Statistics and Informatics from the Bucharest University of Economic Studies in 2007. He has a master in Informatics Project Management (2010) and a $\mathrm{PhD}$ in Economic Informatics (2011) from the Bucharest University of Economic Studies. Cristian has a solid background in computer science and is interested in collaborative systems related issues. Other fields of interest include software metrics, data structures, object oriented programming, windows applications programming, mobile devices programming and testing process automation for software quality assurance.

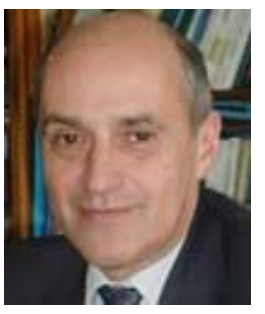

Florin Gheorghe FILIP took his MSc and PhD in control engineering from the TU "Politehnica" of Bucharest. In 1991 He was elected as a member of the Romanian Academy (RA). He has been a scientific researcher at the National R\&D Institute in Informatics (ICI) of Bucharest. Currently he is a part-time researcher at the National Institute of Economic Researches (INCE) of the RA, also the director of the Library of the Academy. He was elected as vicepresident of RA in 2000 and reelected in 2002 and 2006. His main scientific interests include large-scale systems, decision support systems, technology management and foresight. 